\title{
¿Quién sobrevivirá? Algunas consideraciones sobre la oferta de formación en Psicología
}

Juan Martín Sánchez Bautista, ${ }^{1}$

Silvia del Carmen Miramontes Zapata ${ }^{1}$ y Sandra Ramos Basurto ${ }^{1}$

\section{Introducción}

Hace ya dos décadas, Herrera (1993) apuntaba la importancia crucial que tendría la psicología en el futuro, es decir, el presente. En su texto, puntualizaba algunas razones que hacían o hacen de la Psicología una disciplina imprescindible para la sociedad contemporánea, a saber: a) su potencialidad para resolver problemas a nivel familiar, individual, microgrupos y macrogrupos; b) su naturaleza interdisciplinaria; c) su objeto de estudio (el comportamiento humano) y d) su desarrollo científico y profesional. Recordemos que tanto esa década como la siguiente fueron relevantes para la disciplina, la de 1990 fue denominada "década del cerebro", y la siguiente "década de la conducta"; sin embargo, la realidad parece responder a esas consideraciones de una manera totalmente distinta a como se esperaba; en primer lugar, dado que el mercado de trabajo nunca responde a la autovaloración de una profesión en función de: a) cómo es perci-

1 Universidad Autónoma de Zacatecas. 
bida por los propios profesionistas y b) de la necesidad social en función de los problemas que pudiera resolver. De este modo, el planteamiento de Herrera no deja de ser sino una visión romántica sobre por qué una profesión debe existir.

En segundo lugar, el mercado laboral suele ser imperfecto (Mungaray, 2001: 4) y esa:

[...] imperfección se origina porque la formación a través de la educación superior es un proceso lento y tradicionalmente independiente del mundo laboral, y en su toma de decisiones incorpora información que será validada algunos años después. El amplio retraso de tiempo entre selección, educación y entrega de los profesionales al mercado laboral genera desfases de las calificaciones e incertidumbre respecto a la utilidad de estas inversiones.

Recientemente, Ibarra (2013) ha señalado los patrones de contratación de egresados de las Instituciones de Educación Superior (IES) en el país, pues:

[...del] universo total de profesionistas que se producen por año en la República, se pueden mencionar los siguientes componentes descritos por la Subsecretaría de Educación Superior de la SEP: 1) sólo $40 \%$ de ellos ejerce labores en el ámbito de su formación; 2) $60 \%$ no se desempeña en el campo de su profesión; 3) un alto porcentaje egresa con conocimientos obsoletos; y 4) las tasas de desempleo y subempleo son muy elevadas, en consecuencia.

Lo anterior nos enfrenta a una problemática difícil de resolver. El objetivo del presente texto es presentar un panorama del crecimiento de la oferta de formación de psicólogos y señalar algunos riesgos que esto conlleva. 


\section{El camino a la crisis}

Evolución y crecimiento de la oferta de formación en Psicología

Los cuestionamientos o preocupaciones sobre la empleabilidad de la profesión no son nuevos. Pick y Givaudan (1999) tenían una reflexión parecida a la de Herrera (1993) y, en ese sentido, hablaban de la amplitud del campo laboral posible para los psicólogos; sin embargo, las mismas autoras, al considerar el trabajo existente en ese momento, señalaban que por lo menos $50 \%$ de los egresados no tenían empleo. Mirando retrospectivamente, el número de programas que ofrecía la carrera para el año 2000 era de 183, si esto lo contrastamos con la oferta actual de más de 800 programas, tenemos poco qué decir: más de cuatro veces que hace 14 años. Este crecimiento en la oferta de formación, además, ha tenido también un crecimiento en la matrícula y, en consecuencia, ha tenido como efecto una sobreoferta de estos profesionales en el mercado laboral.

¿A qué podemos atribuir esta "crisis" de la profesión? Varios autores han señalado que el número de programas de enseñanza y formación en Psicología como profesión han tenido un crecimiento explosivo y generalmente desordenado (Millan, 1982; Pick y Givaudan, 1999; Zanatta y Yurén, 2012).

Si se hace una revisión cronológica de la enseñanza de la profesión en nuestro país, quizá podamos hacernos una idea sobre el momento o momentos en que se ha generado esta situación. La enseñanza de la Psicología lleva más de un siglo implantada en nuestro país. Es en 1896 que se establece la primera cátedra de Psicología en la Universidad Nacional Autónoma de México (UNAM) y es en el año de 1928 cuando se comienzan a impartir estudios de licenciatura en la misma casa de estudios. Dicho programa se mantuvo como la única opción de formación profesional en esta disciplina hasta la creación de la carrera de Psicología en la Universidad Iberoamericana en 1950, misma que tuvo reconocimiento oficial por su incorporación a la UNAM (Pick y Givaudan, 1999; Zanatta y Yurén, 2012). 
Se puede considerar que en esos primeros años, el crecimiento de las opciones de formación fue relativamente lento, de tal suerte que el número de escuelas y/o programas donde se impartía esta carrera pasó a ser de 10 en 1967, es decir, casi un programa por año. Este lento crecimiento se vio súbitamente "alterado" durante la década de 1970 y se pasó a 25 programas en 1974, 40 en 1977; en otras palabras, el incremento de programas fue un promedio de tres por año durante 1970.

El incremento siguió en la década siguiente, de tal manera que ya se contaba con 66 programas en 1981 y para 1984 ya eran 95; en siete años la oferta incrementó más del doble (Preciado y Rojas, 1989; Galindo y Vorwerg, 1985; Mouret y Ribes, 1977). En la década de 1990, el crecimiento continuó de tal manera que para 1995 ya existían 113 programas, número que ascendió a 147 en 1997, y a partir de ahí hubo un crecimiento constante, hasta llegar a 183 programas en el año 2000, fin de siglo.

El nuevo siglo no mostró cambios sorprendentes en los procesos de crecimiento y del mismo modo se continuó el aumento de la oferta educativa, lo que llevó a que en el año 2005 se contabilizaran 290 programas, es decir, en menos de cinco años más de 100 programas. Si a alguien le sorprende este dato, habrá que señalar que el crecimiento aparece aún más caótico y desordenado en los siguientes años, de tal manera que para 2012 existían alrededor de 800 programas.

\section{Situación actual}

El proceso de crecimiento ocurrido en los últimos siete años ha llevado a que en la actualidad existan, como se ha señalado, más de 800 programas registrados en la SEP. Es pertinente señalar a este respecto que no existe un padrón unificado que permita saber con exactitud la cantidad de programas e instituciones que ofrecen estos estudios, ya que los datos obtenidos en distintos portales web de la SEP y de organismos académicos arrojan un panorama que muestra una oscilación de entre 749 (www.decidetucarrera.ses.sep.gob.mx) y 817 programas con RVOE (http://www.sirvoes.sep.gob.mx/sirvoes/). Además, en los padro- 
nes existentes habría que considerar aquellos programas que se encuentran en trámite de RVOE, así como dejar de computar programas que se encuentran en extinción; sin embargo, para este ejercicio asumiremos el dato mayor que se ha obtenido.

Este gran crecimiento ocurrido durante los últimos años no ha garantizado un aumento de la calidad en la formación que reciben los estudiantes, ni tampoco la garantía en algunos casos de que el centro en el que inician sus estudios les permita concluir éstos.

Un problema al que se enfrenta, además, la enseñanza de la Psicología en las Instituciones de Educación Superior es la continua exigencia de evaluaciones que llevan a categorizar los programas de acuerdo a diferentes parámetros de calidad, lo que puede traer como consecuencia que tengamos, además, estudiantes y profesionistas de diferentes "clases" de acuerdo a la institución de donde egresa, dado que no todas las instituciones y escuelas se someten a estos procedimientos de evaluación o acreditación. El panorama bajo estos criterios es el siguiente: de la oferta actual, solamente 53 programas aparecen como acreditados por los cIIEs (http://www.ciees.edu.mx) en una revisión de 2014, 30 en el Padrón de Licenciaturas de Alto rendimiento del año 2012-2013 (http://idap.ceneval.edu.mx/portal_idap/principal.jsf), mismo que aglutina tanto instituciones públicas como privadas. Y en el mismo sentido, sólo 81 programas aparecen como acreditados por el cNEIP (http://www.cneip.org) para marzo de 2014. Si consideramos la cantidad de programas existentes y el porcentaje de ellos que aparecen en estos distintos padrones, parece que la oferta es desigual en calidad y condiciones de aprendizaje.

\section{Conclusiones}

Se vuelve necesario continuar con las interrogantes y esto hace necesario cuestionarse lo siguiente: ¿Hacia dónde se dirige la formación de psicólogos? En este proceso de crecimiento, al parecer sin proyecto, parece que la formación en la profesión está abocada a una carrera hacia el vacío. Más allá de los discursos de la pertinencia de formar profesionistas, quizá se deba hacer 
una pausa y considerar si con este ritmo de incremento en la oferta de formación del psicólogo no se condena de antemano a los estudiantes al desempleo y en el mejor de los casos al subempleo. Martínez et al. (2009: 34) han señalado que: "Hablar de calidad en la formación de recursos humanos supone un proceso de planeación eficiente", y parece que en ello no estamos. Pensemos si la planeación eficiente de una IES debe contemplar hacia dónde se dirigen nuestros egresados y es que, como señala Tünnermann (2000: 184): "Únicamente un sistema de educación superior suficientemente flexible puede enfrentar adecuadamente los retos de un mercado de trabajo que cambia tan rápidamente". La cuestión final es sobre cómo se decide aumentar la matrícula o centro de formación en las instituciones. Las instituciones formadoras de psicólogos enfrentan un gran reto ya que, por un lado, es impensable que éstas dejen de formar profesionistas; sin embargo, formar recursos humanos que tienen grandes posibilidades de desempleo no parece seguir siendo una opción por las consecuencias sociales y las implicaciones emocionales que tiene sobre los egresados.

\section{Referencias}

Galindo, E. y Vorwerg, M. (1985). La Psicología en México, Ciencia y Desarrollo, 63.

Garrocho Rangel, C. y Segura Lazcano, G. (2012). La pertinencia social y la investigación científica en la universidad pública mexicana (pp. 24-34), CIENCIA ergo sum, Vol. 19-1, marzo-junio 2012.

Herrera, M.A. (1993). La formación profesional del psicólogo. Líneas de trabajo para la configuración de un proyecto alternativo de profesión. Perfiles Educativos, http://www.redalyc.org/articulo.oa?id=13205904.

Ibarra Santos, M. (2013). Las profesiones, mercado laboral y economía del conocimiento en Zacatecas. El Sol de Zacatecas, 14 de marzo de 2013, http://www.oem.com.mx/ elsoldezacatecas/notas/n2913115.htm. 
Martínez-Vargas, S.L., Osorio-García, E., Contreras-Lara-Vega, M.E.A., Solís-Segura, L.M. (2009). Pertinencia social de los egresados del programa educativo de Ingeniero Químico de la Facultad de Química de la Universidad Autónoma del Estado de México. Tecnología, Ciencia, Educación, 24(1), 32-47. Instituto Mexicano de Ingenieros Químicos, México. Disponible en: http://redalyc.uaemex.mx/src/inicio/ ArtPdfRed.jsp?iCve=48212169006.

Millán, P. (1982). La psicología mexicana: una profesión en crisis (49-92). Revista de Educación Superior, 43.

Mouret, E. y Ribes, E. (1977). Panorámica de la enseñanza de la psicología en México. En: Enseñanza e Investigación en Psicología, 3(2), 6-20.

Mungaray, A. (2001). La educación superior y el mercado de trabajo profesional. Revista Electrónica de Investigación Educativa, 3(1). Consultado el día 10 de abril de 2013 en: http://redie.uabc.mx/vol3no1/contenido-mungaray.html.

Ortega Z., M. y Méndez C., C. (2009). La formación del psicólogo educativo y su campo laboral. En Villalobos, G. (Coord.), La carrera de Psicología en la región centro sur de la ANUIES: formación, competencias profesionales e inserción laboral, UAEM.

Pick, S. y Givaudan, M. (1999). La Psicología en México (195215). En Alonso, M. y Eagly, A. (Eds.), Psicología en las Américas. Sociedad Interamericana de Psicología.

Preciado, H. y Rojas, L. (1989). Notas sobre la enseñanza de la Psicologia en México: estado actual y perspectivas de desarrollo. Revista de la Educación Superior, XXI(4), 72. Recuperado de: http://201.161.2.34/servicios/p_anuies/publicaciones/revsup/res072/art5.htm.

Tünnermann, C. (2000). Pertinencia social y principios básicos para orientar el diseño de políticas de educación superior (181-196). Educación Superior y Sociedad, 11(1-2).

Villalobos, G. y Pedroza, R. (2009). Trayectorias vitales e inserción laboral de los egresados de Psicología de la Universidad Autónoma de Tlaxcala y de la Benemérita Universidad Autónoma de Puebla, generación 1998-2005. En Villalo- 
bos, G. (Coord.), La carrera de Psicología en la región centro sur de la ANUIES: formación, competencias profesionales e inserción laboral. UAEM.

Zanatta, E. y Yurén, T. (2012). La formación profesional del psicólogo en México: trayecto de la construcción de su identidad disciplinar, Enseñanza e Investigación en Psicología, 17(1). 